\title{
Методики исследования электрического контактного сопротивления в структуре металлическая пленка-полупроводник
}

\author{
(C) М.Ю. Штерн ${ }^{1}$, И.С. Караваев ${ }^{2}$, М.С. Рогачев ${ }^{1, \uparrow}$, Ю.И. Штерн ${ }^{1}$, Б.Р. Мустафоев ${ }^{1}$, \\ Е.П. Корчагин ${ }^{1}$, А.О. Козлов ${ }^{1}$ \\ ${ }^{1}$ Национальный исследовательский университет „МИЭТ“, \\ 124498 Москва, Зеленоград, Россия \\ ${ }^{2}$ Акционерное общество „Чепецкий механический завод“, \\ 427622 Глазов, Россия \\ IE-mail: m.s.rogachev88@gmail.com
}

Поступила в Редакцию 19 сентября 2021 г.

В окончательной редакции 24 сентября 2021 г.

Принята к публикации 24 сентября 2021 г.

В термоэлементах электрическое сопротивление контактов существенным образом влияет на их эффективность. В случае высоколегированных термоэлектрических материалов на границе контакта металлполупроводник преобладает туннельный механизм проводимости, что позволяет получать сопротивление контакта $<10^{-8} \mathrm{OM} \cdot \mathrm{M}^{2}$. Низкие значения сопротивления существенно усложняют его экспериментальное определение. Представлены три методики и измерительный стенд для исследования контактного сопротивления. Основу методик составляет измерение суммарного электрического сопротивления, состоящего из переходного контактного сопротивления и сопротивления термоэлектрического материала с последующим его исключением. Разработанные методики отличаются расположением исследуемых контактов на образцах термоэлектрических материалов, способами измерений и обработки полученных результатов и позволяют определять удельное контактное сопротивление $\sim 10^{-10} \mathrm{OM} \cdot \mathrm{M}^{2}$.

Ключевые слова: термоэлементы, пленочные контакты, контактное сопротивление, методики измерений.

DOI: 10.21883/FTP.2022.01.51808.24

\section{1. Введение}

При формировании пленочных контактов в полупроводниковых структурах одной из основных характеристик является удельное контактное сопротивление, которое значительно влияет на их эффективность. В термоэлементах значение этого параметра особенно критично, так как термоэлектрические материалы (ТЭМ), из которых изготавливаются термоэлементы, обладают высокой электропроводностью. Концентрация носителей заряда в ТЭМ составляет, как правило, $10^{19}-10^{20} \mathrm{~cm}^{-3}$. В этом случае удельный вес контактного сопротивления может оказаться определяющим в структуре термоэлемента и существенным образом повлиять на его тепло- и электрофизические параметры. Поэтому в технологии термоэлементов особое место принадлежит получению омических контактов. Под омическими контактами понимают контакт металл-полупроводник, сопротивление которого пренебрежимо мало по сравнению с объемным сопротивлением полупроводника при любом направлении тока. Как утверждает автор работы [1], омический контакт должен иметь удельное сопротивление $<10^{-7} \mathrm{OM} \cdot \mathrm{M}^{2}$. В случае контакта к ТЭМ необходимо, чтобы эта величина была не более $10^{-8} \mathrm{OM} \cdot \mathrm{M}^{2}$ [2-6]. Значительное влияние сопротивления контактов на эффективность термоэлектрических устройств отмечают ряд авторов [2-9]. Автор работы [10] утверждает, что при увеличении контактного сопротивления в термоэлементах, работающих на эффекте Пельтье, до 1\% от сопротивления термоэлемента, разность температур (основной его параметр) снижается до $30 \%$ от максимального значения. В работе [11] установлено, что максимальное значение кпд в генераторных термоэлементах может быть получено при удельном сопротивлении контактов на уровне $10^{-10} \mathrm{OM} \cdot \mathrm{M}^{2}$.

Необходимым условием существования омического контакта на границе металл-полупроводник является низкое значение барьера Шоттки. С барьером Шоттки связаны два механизма проводимости: термоэлектронная эмиссия и туннелирование [12-14]. Этим механизмам соответственно отвечают два эмпирических способа изготовления контактов. Первый заключается в выборе металла (по работе выхода), который образует с полупроводником низкий барьер Шоттки. Омические контакты можно получить при использовании металлов с работой выхода, меньшей, чем у полупроводника $n$-типа, и большей, чем у полупроводника $p$-типа. Однако удовлетворяющих этому условию комбинаций металлполупроводник очень мало. В то же время экспериментально для большинства полупроводников было обнаружено, что энергетический барьер не зависит от работы выхода металла, а определяется плотностью поверхностных состояний $[1,12]$. Поэтому широко используемый способ изготовления омических контактов - сильное легирование полупроводника у контакта, достаточное для того, чтобы обеспечить туннелирование носителей тока через барьер. Преобладающая туннельная ком- 
понента тока экспоненциально зависит от концентрации свободных носителей тока [12]. При концентрации $\sim 10^{19} \mathrm{~cm}^{-3}$ сопротивление контакта определяется в основном туннельными процессами, что характерно для ТЭМ.

Низкие значения сопротивления контакта в структуре полупроводник - металлический контакт значительно усложняют его экспериментальное определение. В данной работе предлагается метод измерения контактного сопротивления, аналогичный известному методу TLM (Transmission line method) [8,15-18]. Измеряется суммарное электрическое сопротивление, состоящее из контактного сопротивления и сопротивления подложки, с последующим его исключением. В нашем случае основой являются образцы ТЭМ, на которых ионноплазменным напылением формируются металлические контакты.

Как показали проведенные в данной работе исследования, способы реализации указанного метода для измерения низких контактных сопротивлений (методики измерений) имеют существенное значение для получения стабильных данных с минимальными погрешностями измерений. Предложенные в данной работе методики отличаются расположением исследуемых контактов на образцах ТЭМ, способами измерений и обработки полученных результатов.

Таким образом, цель данной работы заключается в разработке и исследовании экспериментальных методик измерения контактного сопротивления металлических пленок, формируемых на полупроводниковых материалах, в данном случае ТЭМ, а также в сравнительном анализ полученных с помощью этих методик данных для оптимизации измерений.

\section{2. Экспериментальная часть}

\section{1. Подготовка образцов}

В процессе разработки методик измерения контактного сопротивления использовались никелевые контакты, сформированные ионно-плазменным напылением на образцах $\mathrm{Bi}_{2} \mathrm{Te}_{2.8} \mathrm{Se}_{0.2}$, легированного $0.14 \mathrm{Bec} \% \mathrm{CdCl}_{2}$, полученного зонной плавкой.

Состояние поверхности ТЭМ существенным образом влияет на характеристики контактов [17,19-22]. Перед нанесением тонких пленок Ni производилась механическая обработка поверхности образцов ТЭМ до шероховатости 150 нм с последующей очисткой, способы которых представлены в [20]. Шероховатость поверхности образцов и толщина пленки измерялись с помощью профилометра KLA-Tencor P-7.

Перед загрузкой в камеру напылительной системы образцы промывались в изопропиловом спирте с последующей сушкой азотом. Формирование контактов осуществлялось с использованием высоковакуумной системы напыления Angstrom EvoVac 34. Непосредственно в камере производился вакуумно-термический отжиг образцов ТЭМ при начальном давлении $7 \cdot 10^{-8}$ Торр и температуре $200^{\circ} \mathrm{C}$. После отжига проводилась очистка поверхности образцов бомбардировкой ионами аргона в течение 30 с. В процессе вакуумно-термического отжига при помощи квадруполя SRS RGA 200, входящего в состав напылительной системы, контролировалось качество финишной очистки поверхности образцов.

Ионно-плазменное напыление никелевых контактов толщиной 300 нм производилось после ионной очистки и достижении в камере рабочего давления. Режимы напыления были следующие: давление в камере $7 \cdot 10^{-8}$ Торр, скорость напыления $-2 \AA / c$, давление газа $(\mathrm{Ar})-2 \cdot 10^{-3}$, температура $-200^{\circ} \mathrm{C}$.

Формирование контактных площадок на боковых поверхностях образцов ТЭМ осуществлялось с помощью масок, изготовленных из фольги алюминиевого сплава толщиной $0.1 \mathrm{MM}$, в которой на лазерной установке БэтаМАРК 2000 формировались окна диаметром 1.5 мм. Расстояние между контактами определялось с точностью 50 мкм.

Для каждой из методик подготавливались образцы ТЭМ определенных формы и расположения исследуемых контактов. Образцы №1 изготавливались в виде цилиндра длиной 30 мм и диаметром 10 мм. Для нанесения контактов проводилась механическая обработка боковой поверхности образцов вдоль направления роста кристалла. На полученной плоскости через каждые 5.0 мм напылением формировались контактные площадки $\mathrm{Ni}$ диаметром 1.5 мм, к которым припаивались токовые и потенциальные контактные провода.

Образцы №2 изготавливались в виде дисков диаметром 20 мм и толщиной 2 мм. Резка этих образцов проводилась в направлении, перпендикулярном росту кристалла. После этого выполнялась механическая обработка поверхности образцов, на которой через каждые 5 мм формировались никелевые контактные площадки диаметром 1.5 мм. Коммутация токовых и потенциальных проводов к контактным площадкам осуществлялась пайкой.

Образцы №3 изготавливались аналогично образцам №1, но дополнительно на противоположных торцах образцов ТЭМ ионно-плазменным напылением формировались контактные площадки из $\mathrm{Ni}$ толщиной 300 нм. К контактным площадкам на торцах припаивались пластины из $\mathrm{Ni}$ толщиной 1 мм. К контактным площадкам, сформированным на боковой поверхности образца, припаивались коммутационные потенциальные провода для измерения падения напряжения.

Образцы №4 изготавливались в виде цилиндра длиной 20 мм и диаметром 10 мм. Боковая поверхность и торцы образцов механически обрабатывались до шероховатости 150 нм. На противоположных торцах образца ионноплазменным напылением формировались контактные площадки из $\mathrm{Ni}$, к которым припаивались никелевые пластины толщиной 1 мм. 


\section{2. Методики измерений}

Для реализации методик измерения контактного сопротивления разработан стенд, в состав которого входят: измерительная ячейка, оснащенная микрометрическими измерительными индикаторами и цифровым микроскопом с блоком видеоконтроля; мультиметр Keithley 2001 (M); высокоточный электронный термометр ТЭН-4; программируемый источник питания Motech LPS-305 (A) со встроенными амперметром и вольтметром; высокоточный магазин сопротивлений Time Electronics 1067 (RB). Измеренные данные с мультиметра, термометра и микроскопа поступают на компьютер, входящий в структуру стенда.

При малых сопротивлениях, к которым относится исследуемое контактное сопротивление структуры контакт - ТЭМ, в измерительной цепи для источника питания получается практически короткое замыкание. В этом случае большинство источников не способно с приемлемой точностью стабилизировать заданный ток. Поэтому последовательно к образцу в измерительную цепь включен магазин сопротивлений. Регулированием параметров источника питания и магазина сопротивлений в цепи устанавливалось необходимое значение тока, которое стабилизировалось с погрешностью, не превышающей $0.1 \%$.

В процессе измерений на образце ТЭМ контролировался профиль температур с помощью двух установленных в токовых зондах тонкопленочных платиновых терморезисторов Pt-1000. Терморезисторы подключались к термометру ТЭН-4 с погрешностью измерений $0.05^{\circ} \mathrm{C}$. Погрешность измерения напряжения с учетом чувствительности мультиметра $\left(10^{-7} \mathrm{~B}\right)$ не превышала $0.2 \%$. Необходимо отметить, что при измерениях падения напряжения на исследуемых структурах использовалась четырехпроводная схема, позволяющая устранить погрешности, определяемые сопротивлением подводящих проводов. Ток через образец пропускался в двух противоположных направлениях. Изменение направления постоянного тока необходимо для устранения влияния термоэлектрических эффектов на результаты измерений.

Конструкция измерительной ячейки представлена на рис. 1. Исследуемый образец ТЭМ (1) устанавливался между токовыми зондами (2), в медной оснастке (3), через слюду толщиной 20 мкм. Это позволяет минимизировать градиент температуры на образце в процессе измерений. Площадь контакта токовых зондов должна быть не меньше поперечного сечения исследуемых образцов. Медная оснастка с образцом закреплялась на основании (4), перемещение которой относительно измерительного зонда (5) регулировалось с помощью прецизионного механизма (6). Таким образом регулировался шаг сканирования зонда. Измерительный зонд изготовлен из стальной иглы с золотым покрытием. Усилие нагрузки на образец ТЭМ, размещенный между токовыми зондами, определялось с помощью устройства (7). Для контроля перемещения основания с образцом и,

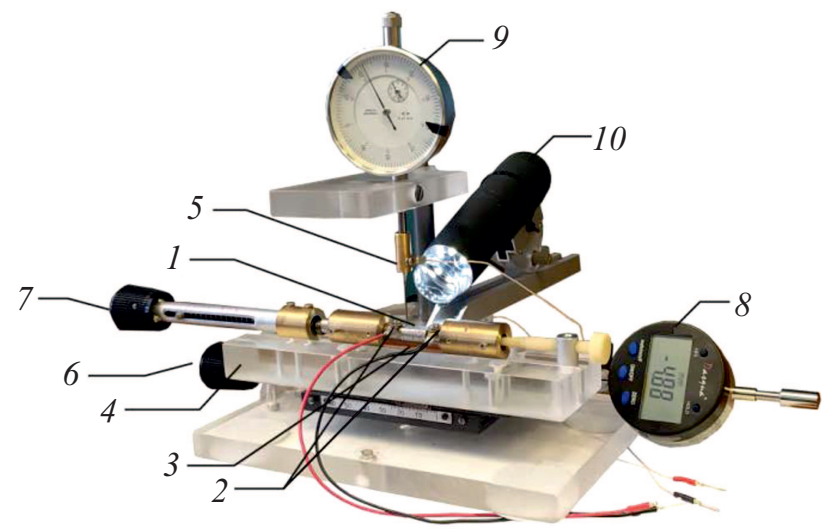

Рис. 1. Измерительная ячейка для определения контактного сопротивления.

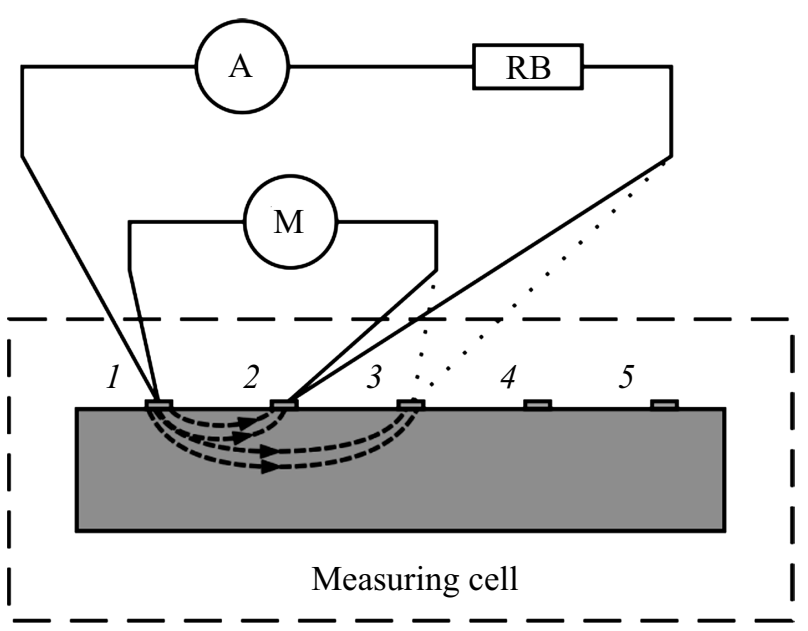

Рис. 2. Схемы образцов №1 и 2 и измерения контактного сопротивления по первой методике.

соответственно, шага сканирования измерительного зонда использовался микрометрический индикатор (8). Положение зонда определялось с погрешностью 10 мкм. Усилие прижима измерительного зонда к исследуемому образцу контролировалось с помощью откалиброванного микрометрического измерительного индикатора (9). Для визуализации процесса измерений использовался цифровой микроскоп (10), соединенный с ПК.

Первая методика измерения. В первой методике для измерения контактного сопротивления использовались образцы №1 и 2. На рис. 2 представлена схема измерения контактного сопротивления. Цифрами обозначены номера контактных площадок на боковой поверхности образца. Как было показано выше, при измерениях падения напряжения на исследуемых структурах использовалась четырехпроводная схема. При измерении контактного сопротивления исследуемый образец устанавливался в измерительную ячейку и фиксировался между токовыми зондами. В первой методике эти зонды использовались только для крепления исследуемого 
образца в ячейке и измерения температуры на нем. Ток подавался на образец с помощью токовых проводов, припаянных к контактным площадкам. Ток, значением $100 \mathrm{MA}$, от источника питания (А) пропускался между контактной площадкой №1 и последовательно контактными площадками №2-5. Падение напряжения измерялось с помощью потенциальных проводов мультиметром (М). Измерения проводились при противоположных направлениях тока.

Вторая методика измерения. Схемы измерения контактного сопротивления по второй методике и размещения контактов на образце представлены на рис. 3.

При измерении контактного сопротивления по второй методике использовался образец ТЭМ №3, который устанавливался в измерительную ячейку между токовыми зондами. С помощью зондов, подключенных к источнику питания, через образец пропускался ток $100 \mathrm{MA}$. Падение напряжения определялось с помощью потенциальных проводов между первой контактной площадкой и последовательно остальными контактными площадками, сформированными на боковой поверхности. Измерения проводились при противоположных направлениях тока.

Третья методика измерения. Для исследования контактного сопротивления по третьей методике использовался образец №4. Схема измерения контактного

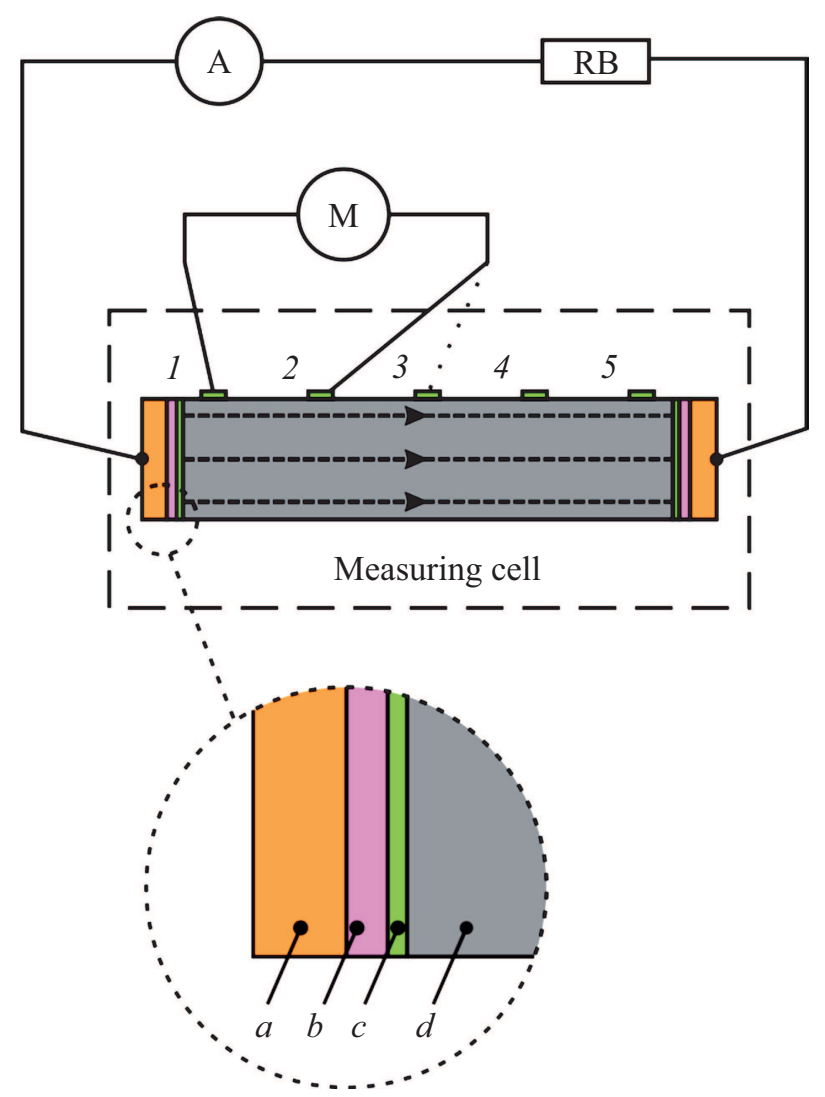

Рис. 3. Схемы образца №3 и измерения контактного сопротивления по второй методике. $a-\mathrm{Ni}$-пластина, $b-$ припой, $c$ - никелевая контактная площадка, $d-$ образец ТЭМ.

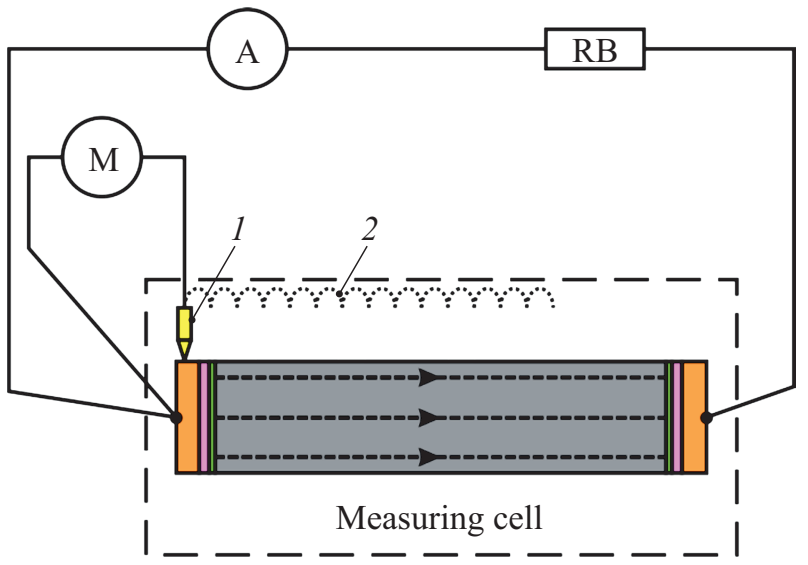

Рис. 4. Схема измерения контактного сопротивления по третьей методике. 1 - измерительный зонд, 2 - траектория движения измерительного зонда.

сопротивления представлена на рис. 4. Образец помещают в измерительную ячейку между токовыми зондами. Перемещение образца и, соответственно, сканирование измерительного зонда по боковой поверхности образца осуществлялось с шагом от 50 до 500 мкм и контролировалось с помощью микрометрического индикатора. Измерение проводилось при сканировании измерительного зонда по всей длине образца ТЭМ от одной никелевой пластины на торце образца до другой. После установки измерительного зонда, через образец посредством токовых зондов с источника питания пропускали ток $300 \mathrm{MA}$. В каждой точке на поверхности образца, определяемой шагом сканирования, проводили по два измерения падения напряжения между торцевой никелевой пластиной и измерительным зондом при противоположных направлениях тока.

\section{3. Результаты исследования и обсуждение}

Для определения контактного сопротивления исследуемых структур по первой и второй методикам сначала рассчитывалось сопротивление структуры по формуле

$$
R=U / I,
$$

где $U$ - падение напряжения, $I$ - ток.

При измерениях ток пропускался в двух направлениях, а падение напряжения на структуре определялось по формуле

$$
U=\left(\left|U_{1}\right|+\left|U_{2}\right|\right) / 2,
$$

где $U_{1}$ и $U_{2}$ - падения напряжений при пропускании тока в двух противоположных направлениях.

Контактное сопротивление рассчитывалось следующим образом. Измерялись сопротивления, например, $R_{s t r 12}$ и $R_{s t r 13}$. Сопротивление $R_{s t r 12}$ между контактными площадками 1 и 2 (рис. 2 и 3) включает сопротивления 
Результаты исследования контактного сопротивления

\begin{tabular}{c|c|c|r|c|c|c|c}
\hline $\begin{array}{c}\text { № } \\
\text { образца }\end{array}$ & $I, \mathrm{MA}$ & $\begin{array}{r}U_{12}, \\
\text { мкВ }\end{array}$ & $\begin{array}{r}U_{13}, \\
\text { мкВ }\end{array}$ & $\begin{array}{c}R_{\text {str } 12,}, \\
\text { мОм }\end{array}$ & $\begin{array}{c}R_{\text {str 13, }}, \\
\text { мОм }\end{array}$ & $\begin{array}{c}R_{c}, \\
\text { мОм }\end{array}$ & $\rho_{c}, \mathrm{OM} \cdot \mathrm{M}^{2}$ \\
\hline 1 & 100 & 644 & 1141 & 6.44 & 11.41 & 0.74 & $1.3 \cdot 10^{-9}$ \\
2 & 100 & 820 & 1502 & 8.20 & 15.02 & 0.69 & $1.2 \cdot 10^{-9}$ \\
3 & 100 & 476 & 641 & 1.59 & 2.14 & 0.51 & $0.9 \cdot 10^{-9}$
\end{tabular}

контактов 1 и $2\left(R_{c}\right)$, а также сопротивление ТЭМ на участке между этими контактами $\left(R_{M 12}\right)$ :

$$
R_{s t r 12}=2 R_{c}+R_{M 12} .
$$

Соответственно $R_{s t r 13}$ будет равно:

$$
R_{s t r 13}=2 R_{c}+R_{M 13} .
$$

Отношение $\mathrm{R}_{M 12}$ и $R_{M 13}$ определяется расстояниями между контактными площадками 1,2 и 1,3 . Так как контакты формируются на одинаковом расстоянии, то

$$
R_{M 13}=2 R_{M 12} .
$$

Тогда сопротивление контакта может быть рассчитано по формуле

$$
R_{c}=\left(2 R_{s t r 12}-R_{\text {str } 13}\right) / 2 .
$$

Удельное контактное сопротивление с учетом площади контактной площадки $\left(S_{c}\right)$ определялось следующим образом:

$$
\rho_{c}=R_{c} S_{c} .
$$

ТЭМ, полученные направленной кристаллизацией, обладают анизотропией свойств, в том числе электропроводности. Поэтому для проверки достоверности полученных результатов представляет интерес измерение контактного сопротивления при пропускании тока вдоль (образец №1) и поперек (образец №2) направления роста кристалла ТЭМ. В лучшем случае, измеренные контактные сопротивления, полученные на обоих образцах, вырезанных из слитков кристалла ТЭМ вдоль и поперек его роста, должны иметь одинаковые значения.

Результаты измерений, полученные при реализации первой методики (образцы №1 и 2) и второй методики (образец №3), представлены в таблице.

Анализируя полученные данные, необходимо отметить следующее: электрическое сопротивление структуры $\left(R_{s t r}\right)$ у образцов №1 и 2 отличаются. Это определяется анизотропией значений электропроводности ТЭМ. В частности, электропроводность ТЭМ вдоль оси роста кристалла выше, чем в перпендикулярном направлении. На контактное сопротивление этот эффект не влияет. Поэтому полученные значения $R_{c}$ и $\rho_{c}$ на образцах №1 и 2 отличаются не больше, чем это определяет погрешность измерений.

Значения контактного сопротивления, определенные по первой и второй методикам, отличаются, что связано, скорее всего, с распределением линий тока при измерениях. С этой точки зрения вторая методика является предпочтительной, так как линии тока в ней распределены равномерно. Влияние распределения тока на точность электрофизических измерений отмечено также авторами $[23,24]$.

Для исследования контактного сопротивления по третьей методике использовался образец №4. Пластина никеля в торцевом контакте у этого образца выполняла две функции. Первая - это площадка для установки измерительного зонда (установить зонд на пленку толщиной 300 нм не представляется возможным). Вторая (как и у образца №3) - это равномерное распределение токовых линий по сечению образца при пропускании через него тока.

В процессе сканирования зонда по поверхности образца измерялось падение напряжения между никелевой пластиной и измерительным зондом. Затем рассчитывалось текущее сопротивление по формуле (1). При исследованиях по этой методике увеличили ток, пропускаемый через образец, до 300 мА. Это необходимо, чтобы компенсировать погрешность, возникающую за счет уменьшения расстояния между точками измерений, что приводит к значительному снижению измеряемого напряжения.

В процессе перемещения зонда по образцу изменялся шаг сканирования. В области измеряемого контакта шаг был 50 мкм, что связано с необходимостью более точного определения его положения. При сканировании зонда по термоэлектрическому материалу такой точности не требуется. Поэтому для оперативности измерений шаг был увеличен до 500 мкм.

Изменение сопротивления при перемещении зонда по образцу схематически показано на рис. 5. Значение сопротивления контактов определялось увеличением измеренного сопротивления при переходе зонда с никелевой пластины на ТЭМ на одном конце образца, или с ТЭМ на никелевую пластину на другом конце образца. Сопротивление контактов определялось следующим образом:

$$
\begin{aligned}
& R_{c 1}=R_{1}-R_{p 1}, \\
& R_{c 2}=R_{p 2}-R_{2},
\end{aligned}
$$

где $R_{p 1}$ и $R_{p 2}$ - измеренные сопротивления на никелевых пластинах. $R_{1}$ и $R_{2}-$ измеренные сопротивления в начале и конце образца ТЭМ соответственно (рис. 5).

Стоит отметить, что при этом в значениях сопротивления контактов присутствует сопротивление ТЭМ. Это определяется тем, что минимальный шаг перемещения измерительного зонда (50 мкм) существенно больше толщины напыленного слоя $\mathrm{Ni}(300 \mathrm{Hм})$. Но это увеличение контактного сопротивления не существенно влияет на результат измерений, потому что определяется небольшой толщиной слоя ТЭМ и высокой его электропроводностью. Кроме того, вклад ТЭМ в сопротивление контакта может быть рассчитан. 


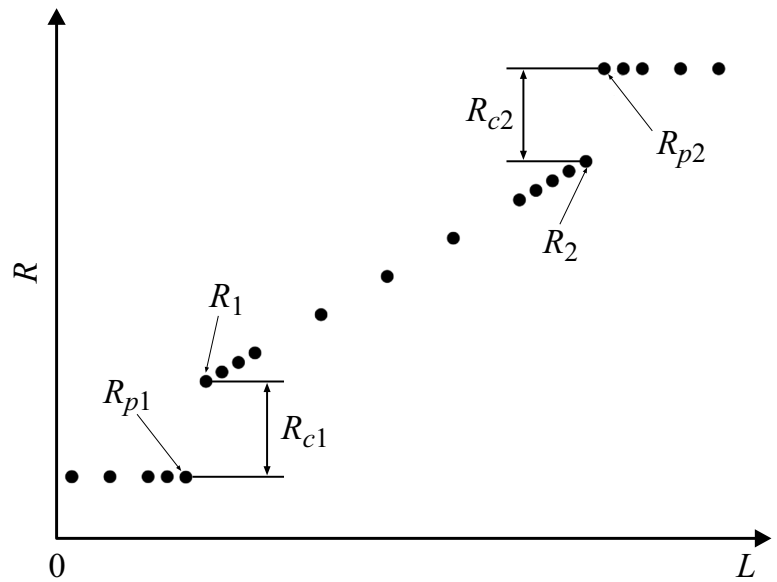

Рис. 5. Схема изменения электрического сопротивления от положения измерительного зонда на образце ТЭМ.

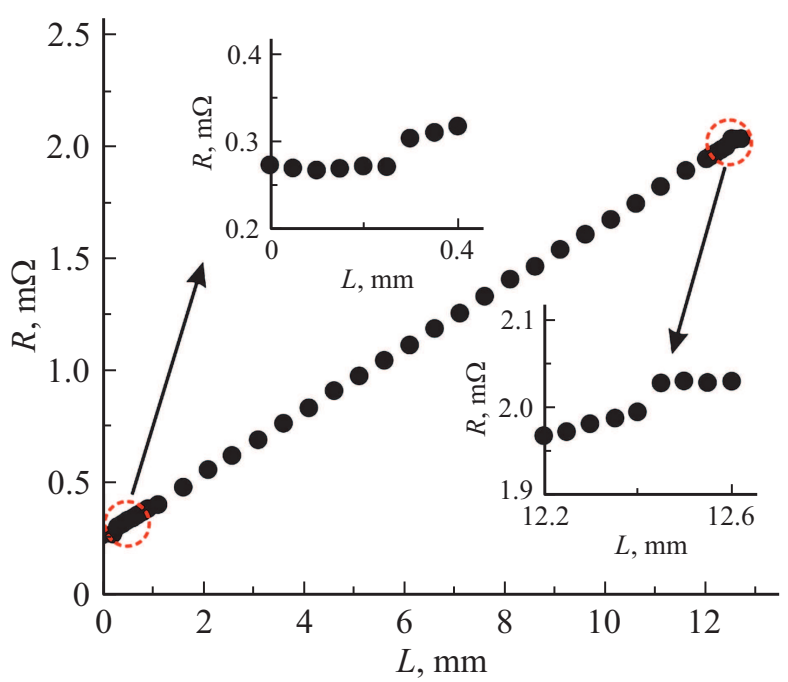

Рис. 6. Зависимость электрического сопротивления на образце №4 от положения измерительного зонда.

В процессе измерений определялось два значения контактного сопротивления на противоположных торцах образца. Среднее значение рассчитывалось следующим образом:

$$
R_{c . a v}=\left(R_{c 1}+R_{c 2}\right) / 2 .
$$

Удельное контактное сопротивление определялось из формулы (7).

Экспериментальные данные исследования контактного сопротивления образца №4 представлены на рис. 6.

При перемещении измерительного зонда на первом участке сопротивление слабо изменяется и определяется сопротивлением никелевой пластины (рис. 6). При переходе измерительного зонда с никелевой пластины на образец ТЭМ наблюдается резкое увеличение сопротивления. Далее при сканировании зонда по поверхности образца сопротивление, определяемое ТЭМ, с расстоянием увеличивается линейно. В конце образца, при переходе зонда на никелевую пластину также наблюдается второе значительное увеличение сопротивления. Резкое изменение сопротивления в начале и в конце образца определяется контактным сопротивлением. Определенное с использованием третьей методики удельное контактное сопротивление составило $2.2 \cdot 10^{-9} \mathrm{OM} \cdot \mathrm{M}^{2}$.

Предложенные методики могут быть использованы для исследований в процессе разработки технологии контактных систем к полупроводниковым материалам, в том числе к ТЭМ, в равной степени. Выбор методики может определяться технологическими возможностями исследователей при формировании контактов.

Как показали исследования, с приемлемой погрешностью можно определять удельное контактное сопротивление $\sim 10^{-10} \mathrm{OM} \cdot \mathrm{M}^{2}$, что свидетельствует о высокой чувствительности методик. Для каждой методики определены погрешности измерений с учетом размеров образцов и выбранных режимов исследования. Для первых двух методик погрешность находится на уровне $3 \cdot 10^{-10}$ и $1 \cdot 10^{-10} \mathrm{OM} \cdot \mathrm{M}^{2}$ соответственно. Для третьей методики погрешность составила $5 \cdot 10^{-10} \mathrm{OM} \cdot \mathrm{M}^{2}$. Большая часть погрешности третьей методики связана с определением положения измерительного зонда и может быть уменьшена, как было показано выше, с помощью расчета.

Полученные результаты хорошо согласуются с литературными данными для качественных тонкопленочных контактов, сформированных вакуумным напылением [16,19,25-27]. Необходимо также отметить, что низкие значения контактного сопротивления свидетельствуют о качестве технологии получения пленочных контактов к ТЭМ, представленных в данной работе.

Стоит отметить, что третья методика измерений может быть использована для определения электропроводности термоэлектрических материалов.

\section{4. Заключение}

Разработаны три методики и измерительный стенд для исследования контактного сопротивления в структуре пленочный металлический контакт-полупроводник. Проведена экспериментальная апробация методик на структурах металлическая пленка - термоэлектрический материал, в которых удельное контактное сопротивление составляет $\sim 10^{-9} \mathrm{OM} \cdot \mathrm{M}^{2}$. Методики отличаются расположением исследуемых контактов на образцах ТЭМ, способами измерений и обработки полученных результатов.

Установлена высокая чувствительность определения контактного сопротивления, которая находится на уровне $10^{-10} \mathrm{OM} \cdot \mathrm{M}^{2}$. Определены погрешности измерений для каждой методики, которые не превышают $5 \cdot 10^{-10} \mathrm{Oм} \cdot \mathrm{M}^{2}$. Предложенные методики могут быть использованы для исследований в процессе разработки технологии контактов к полупроводниковым материалам, в том числе контактов в термоэлементах. Выбор 
одной из методик может определяться технологическими возможностями исследователей при формировании контактов.

\section{Финансирование работы}

Работа выполнена при поддержке Российского научного фонда (проект № 20-19-00494).

\section{Конфликт интересов}

Авторы заявляют, что у них нет конфликта интересов.

\section{Список литературы}

[1] E.H. Rhoderick, R.H. Williams. Metal-Semiconductor Contacts (Oxford, University Press, 1988).

[2] R.P. Gupta, K. Xiong, J.B. White, K. Cho, H.N. Alshareef, B.E. Gnade. J. Electrochem. Soc., 157 (6), H666 (2010).

[3] A. Ferrario, S. Battiston, S. Boldrini, T. Sakamoto, E. Miorin, A. Famengo, A. Miozzo, S. Fiameni, T. Iida, M. Fabrizio. Materials Today: Proceedings, 2 (2), 573 (2015).

[4] M. Shtern, M. Rogachev, Y. Shtern, D. Gromov, A. Kozlov, I. Karavaev. J. Alloys Compd., 852, 156889 (2021).

[5] G. Joshi, D. Mitchell, J. Ruedin, K. Hoover, R. Guzman, M. McAleer, L. Wood, S. Savoy. J. Mater. Chem. C, 7 (3), 479 (2019).

[6] W. Liu, H. Wang, L. Wang, X. Wang, G. Joshi, G. Chen, Z. Ren. J. Mater. Chem. A, 1 (42), 13093 (2013).

[7] T. Sakamoto, Y. Taguchi, T. Kutsuwa, K. Ichimi, S. Kasatani, M. Inada. J. Electron. Mater., 45 (3), 1321 (2016).

[8] Y. Thimont, Q. Lognone, C. Goupil, F. Gascoin, E. Guilmeau. J. Electron. Mater., 43 (6), 2023 (2014).

[9] K. Xiong, W. Wang, H.N. Alshareef, R.P. Gupta, J.B. White, B.E. Gnade, K. Cho. J. Phys. D: Appl. Phys., 43 (11), 115303 (2010).

[10] W. Hanlein. Kaltetechnik, 2, 137 (1960).

[11] W. Liu, Q. Jie, H.S. Kim, Z. Ren. Acta Mater., 87, 357 (2015).

[12] S.M. Sze, K.K. Ng. Physics of Semiconductor Devices (N. Y., Wiley, 2007).

[13] K.K. Ng, R. Liu. IEEE Trans. Electron Dev., 37, 1535 (1990).

[14] Т.В. Бланк, Ю.А. Гольдберг. ФТП, 41 (11), 1281 (2007).

[15] V. Kessler, M. Dehnen, R. Chavez, M. Engenhorst, J. Stoetzel, N. Petermann, K. Hesse, T. Huelser, M. Spree, C. Stiewe, P. Ziolkowski, G. Schierning, R. Schmechel. J. Electron. Mater., 43 (5), 1389 (2014).

[16] D. Qin, W. Zhu, F. Hai, C. Wang, J. Cui, Y. Deng. Adv. Mater. Interfaces, 6 (20), 1900682 (2019).

[17] X. Zhu, L. Cao, W. Zhu, Y. Deng. Adv. Mater. Interfaces, 5 (23), 1801279 (2018).

[18] C.C. Yu, H.-j. Wu, M.T. Agne, I.T. Witting, P.-Y. Deng, G.J. Snyder, J.P. Chu. APL Mater., 7 (1), 013001 (2019).

[19] P.A. Sharma, M. Brumbach, D.P. Adams, J.F. Ihlefeld, A.L. Lima-Sharma, S. Chou, J.D. Sugar, P. Lu, J.R. Michael, D. Ingersoll. AIP Adv., 9 (1), 015125 (2019).

[20] M.Yu. Shtern, I.S. Karavaev, Y.I. Shtern, A.O. Kozlov, M.S. Rogachev. Semiconductors, 53 (13), 1848 (2019).

[21] Е.К. Белоногов, В.А. Дыбов, А.В. Костюченко, С.Б. Кущев, Д.В. Сериков, С.А. Солдатенко. Поверхность. Рентгеновские, синхротронные и нейтронные исследования, 5, 17 (2019).
[22] D. Zillmann, D. Metz, B. Matheis, A. Dietzel, A. Waag, E. Peiner. J. Electron. Mater., 48 (9), 5363 (2019).

[23] А.Т. Бурков, А.И. Федотов, А.А. Касьянов, Р.И. Пантелеев, Т. Накама. Науч.-техн. вестн. информационных технологий, механики и оптики, 15 (2), 173 (2015).

[24] В.И. Смирнов, Ф.Ю. Матта. Теория конструкций контактов в электронной аппаратуре (М., Сов. радио, 1974).

[25] R.P. Gupta, J.B. White, O.D. Iyore, U. Chakrabarti, H.N. Alshareef, B.E. Gnade. Electrochem. Solid-State Lett., 12 (8), H302 (2009).

[26] P.J. Taylor, J.R. Maddux, G. Meissner, R. Venkatasubramanian, G. Bulman, J. Pierce, R. Gupta, J. Bierschenk, C. Caylor, J. D'Angelo. Appl. Phys. Lett., 103 (4), 043902 (2013).

[27] S.-P. Feng, Y.-H. Chang, J. Yang, B. Poudel, B. Yu, Z. Ren, G. Chen. Phys. Chem. Chem. Phys., 15 (18), 6757 (2013).

Редактор А.Н. Смирнов

\section{Methods for investigation of electrical contact resistance in a metal film - semiconductor structure}

M.Yu. Shtern ${ }^{1}$, I.S. Karavaev ${ }^{2}$, M.S. Rogachev ${ }^{1}$, Yu.I. Shtern ${ }^{1}$, B.R. Mustafoev ${ }^{1}$, E.P. Korchagin ${ }^{1}$, A.O. Kozlov ${ }^{1}$

${ }^{1}$ National Research University of Electronic Technology, 124498 Moscow, Russia 2 JSC „Chepetsky Mechanical Plant", 427622 Glazov, Russia

Abstract The electrical contact resistance significantly affects the efficiency of thermoelements. In the case of high doped thermoelectric materials, the tunneling mechanism of conductivity prevails at metal-semiconductor interface, which makes it possible to obtain a contact resistance of less than $10^{-8} \mathrm{Ohm} \cdot \mathrm{m}^{2}$. Low resistance values significantly complicate its experimental determination. Work present three techniques and a measuring stand for the investigation of contact resistance. The techniques are based on the measurement of the total electrical resistance, which consists of transient contact resistance and the resistance of the thermoelectric material with its subsequent exclusion. The developed techniques differ in the arrangement of the investigated contacts on the samples, in the methods of measurement and processing of the obtained results, and make it possible to determine the specific contact resistance of the order of $10^{-10} \mathrm{Ohm} \cdot \mathrm{m}^{2}$. 